$\mathrm{p}=0.02)$. A similar proportion of Black compared to White women had OSA (33\% vs. 31\%). Although severity of OSA was non-significantly elevated in Blacks (AHI 9.2 vs 6.3, $\mathrm{p}=0.07)$, minimum oxygen saturation was significantly lower in Black women $(89 \%$ vs $91 \%, \mathrm{p}=0.04)$ and the oxygen desaturation index was higher in Blacks compared to Whites (4.9 vs 2.5, $\mathrm{p}=0.03)$ after accounting for differences in demographics.

Discussion The incidence of OSA in pregnancy was high with approximately one-third of all women having OSA. Nonetheless, despite being younger and earlier in gestation, Black women had greater severity of oxygen desaturation compared to Whites; this is likely attributed to the higher BMI observed in Black women. These findings have implications for OSA screening in pregnancy.

\section{CHILDHOOD NARCOLEPSY AND AUTISM SPECTRUM DISORDER: A RETROSPECTIVE CASE NOTES REVIEW OF CLINICAL CHARACTERISTICS}

Catherine Jesson*, Rachel Coleman-Smith, Nick Stenning, Heather Elphick. Sheffield Children's Hospital NHS Foundation Trust, Sheffield, UK

\subsection{6/bmjresp-2021-bssconf.38}

Background Autism spectrum disorder (ASD) is often seen alongside narcolepsy in childhood; however, little is known about the potential link between the two. Our objective was to identify any similarities or differences between children with narcolepsy who also have ASD and those who do not.

Methods A single-centre retrospective records review was undertaken of all children attending narcolepsy clinics as of 1st of August 2021. Data collected included: date and method of narcolepsy diagnosis, severity of narcolepsy at diagnosis, Revised Children's Anxiety and Depressions Scale (RCADS) scores from parent and child, presence of autistic traits, date of ASD diagnosis and support received by the child's family.

Results Data was collected from 83 sets of patient records. of this sample, $75(90.4 \%)$ had a confirmed diagnosis of narcolepsy, further analysis was conducted on this group only. A total of $21(28.0 \%)$ children were recorded to have autistic traits, $9(12.0 \%)$ had a confirmed diagnosis of ASD; $88 \%$ of ASD diagnoses were made before investigation for narcolepsy. Children with and without ASD had similar SOL and REMSOP results on MSLT. When collecting RCADS data, $55.6 \%$ of questionnaires from children with ASD were incomplete for both parent and child, compared to $29.6 \%$ of questionnaires from cases without, there was greater discrepancy between parent and child scores in the ASD group and higher parent-rated anxiety scores. Children with ASD were also more likely to receive enhanced school support.

Discussion Descriptive analysis of this sample has shown that $40 \%$ of children with narcolepsy also have and ASD diagnosis or autistic traits. These children were more likely to be rated as anxious by their parents and went on to require enhanced support throughout school. This may suggest that ASD is could act as a clinical indicator to offer enhanced support where possible.

\section{EXPLORING PATTERNS OF BEDTIME BEHAVIOUR IN A COHORT OF CHILDREN WITH SEVERE BEHAVIOURAL INSOMNIA}

${ }^{2}$ Louis Stokes*, 'Lowri Thomas, 'Ruth Kingshott, 'Heather Elphick. 'Sheffield Children's Hospital NHS Foundation Trust, Sheffield, UK; ${ }^{2}$ University of Sheffield, Sheffield, UK

\subsection{6/bmjresp-2021-bssconf.39}

Introduction A child's activities during the hour before bedtime forms the basis of the assessment for behavioural change interventions for children's insomnia. The aim of this observational study was to identify patterns of behaviour around bedtime in a cohort of children with severe sleep difficulties and to explore deviations in behaviour from The Sleep Charity's standard bedtime routine advice.

Methods Data were collected by research staff using a questionnaire during a baseline face-to-face visit to the child's home prior to sleep practitioner support as part of the Sheffield Children's Sleeping Well study. Children were aged 2-17 years with a diagnosis of ADHD or were identified as a looked-after child. Data was input into NVivo where it was coded to identify common or recurring keywords or phrases in the responses.

Results 51 parents were interviewed. Table 1 presents the coded responses to questions relating to sleep behaviours. For 32 children, bedtime routines lasted 30 mins- 2 hours; 17 had no routine. Only 13 children were reported to have actively had technology removed within the hour before bedtime. 32 had no planned snack. of the 17 that did, snacks were usually cereal, toast, biscuit, warm milk although some snacks

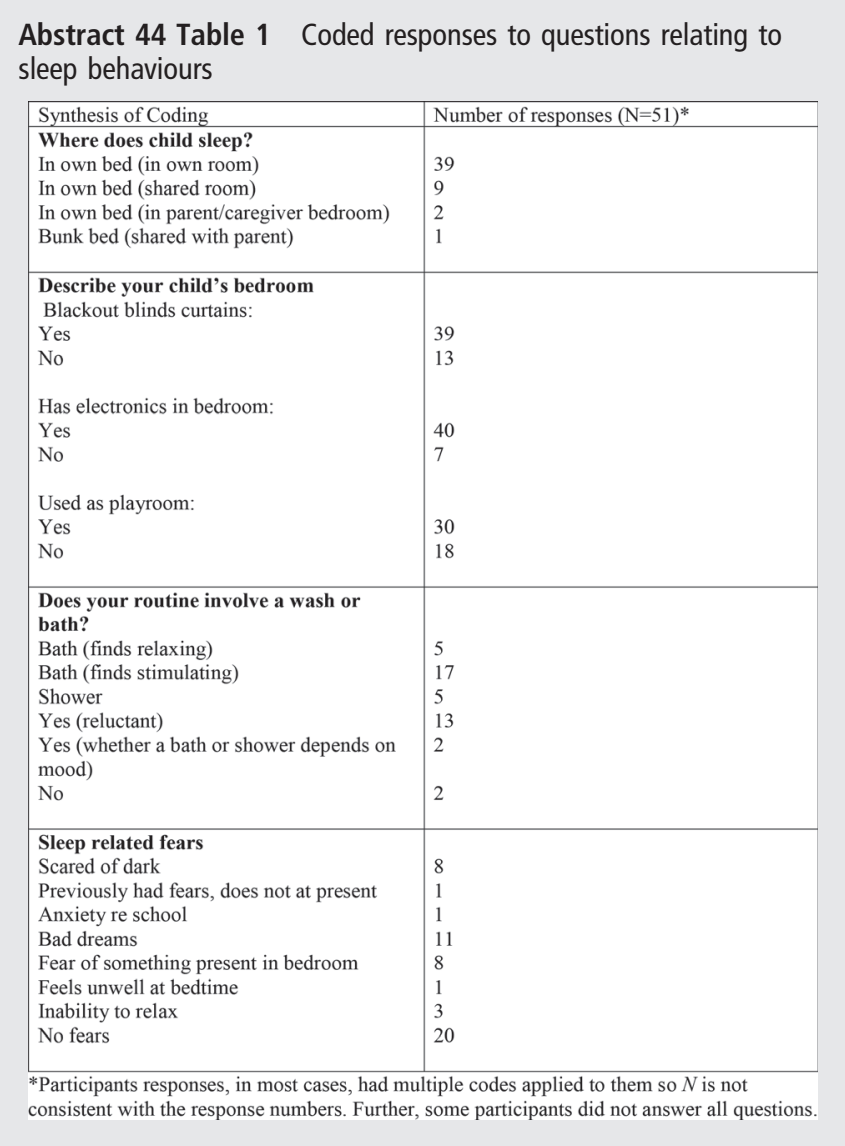


included chocolate, hot chocolate, crisps, burger and chips. Only10 children engaged in calming activities in the hour before bedtime such as reading, colouring, lego and crafts. 28 children were left to self-settle and 22 had a parent laying or sitting next to them in their bedroom in order to fall asleep.

Conclusion There was marked variation from the recommended bedtime routine in children with severe behavioural sleep difficulties, accepting that in some, socially desirable responses may have been given to the research staff during the assessment. Further work to explore the important factors in helping a child to self-settle is ongoing.

\section{THE SLEEP, CIRCADIAN RHYTHMS AND MENTAL HEALTH IN SCHOOLS (SCRAMS) FEASIBILITY STUDY}

${ }^{1}$ Giulia Gaggioni*, ${ }^{1}$ Judith Brown, ${ }^{2}$ Stella Chan, ${ }^{1}$ Breda Cullen, ${ }^{3}$ Michael Farquhar ${ }^{1}$ Daniela Gibbons, ${ }^{4}$ Alice Gregory, ${ }^{1}$ Joanna Inchley, ${ }^{1}$ Laura Lyall, ${ }^{5}$ Mark Matthews, ${ }^{6}$ Alyson O'Brien, ${ }^{1}$ Natasha Sangha, ${ }^{1}$ Sharon Simpson, ${ }^{7}$ Manuel Spitschan, ${ }^{8}$ Malcolm von Schantz, ${ }^{9}$ Heather Whalley, ${ }^{10}$ Cathy Wyse, ${ }^{9}$ Daniel Smith. ${ }^{1}$ Institute of Health and Wellbeing, College of Medical, Veterinary and Life Sciences, University of Glasgow, UK; ${ }^{2}$ School of Psychology and Clinical Language Sciences, University of Reading, United Kindom; ${ }^{3}$ Evelina London Children's Hospital, London, United Kindom; ${ }^{4}$ Department of Psychology, Goldsmiths, University of London, United Kindom; ${ }^{5}$ School of Computer Science, University College Dublin, Ireland; ${ }^{6}$ Sleep Scotland, Edinburgh, United Kindom; ' Department of Experimental Psychology, University of Oxford, United Kindom; ${ }^{8}$ Department of Psychology, Northumbria University, United Kindom; ${ }^{9}$ Centre for Clinical Brain Sciences, University of Edinburgh, United Kindom; ${ }^{10}$ Division of Population Health Sciences, Royal College of Surgeons in Ireland, Dublin, Ireland

\subsection{6/bmjresp-2021-bssconf.40}

Introduction Adolescence is a key developmental period for the onset of mental illness. Healthy rhythms of sleep and activity are critical for mental wellbeing in young people. Yet getting healthy diurnal rhythms becomes challenging for teens, due to developmental changes and multiple social and technological factors modifiable risk factor. We addressed this relationship in a feasibility study by investigating sleep-wake patterns of teenagers and their mental health, wellbeing, and cognitive performance.

Methods 9 schools of the SHINE network in Scotland took part in the study. Pupils wore an actigraph for 3 weeks, allowing the collection of objective rest-activity data. During the 3 weeks, they filled in -twice a day- a brief ecological momentary assessment with mood questions on their mobile phone (EMAapp). At the beginning and at the end of this period, participants also completed a digital online survey with further questions about mental health, sleep, and wellbeing (pre- and post- questionnaire) and they performed a cognitive assessment (6 subtests) on the online platform TestMyBrain (TMB).

Results The feasibility study included two data acquisitions. In Winter (Feb-March 2021), during the lockdown, 8 schools took part for a total of 60 students, whereas, in Summer (May-June 2021), 3 schools took part for a total of 15 students.

Preliminary feasibility results are summarised in the table 1 below.

Discussion Overall, relatively good participation, especially during the lockdown. Compliance decreased from the pre- to the post- questionnaire session and within TMB itself. Only 2 people fully completed the study. $11 \%$ of the pupils had problem with the EMA app, as reflected in the average low entries. Regarding the actigraph, it was generally well tolerated (a few pupils complained having a rash at the end). Further actigraphy analyses are ongoing.

Funding MRC Engagement Award for Adolescent Mental Health.

\section{IS SUBJECTIVE SLEEP CONTINUITY ASSOCIATED WITH FLUID INTELLIGENCE?}

Emily L Jensen*, Nayantara Santhi, Greg J Elder. Northumbria Sleep Research, Northumbria University, Newcastle upon Tyne, UK

\subsection{6/bmjresp-2021-bssconf.41}

Introduction Previous research has indicated that poor subjective sleep quality is associated with lower fluid intelligence scores (Smith et al., 2019). However, it is not known whether subjective sleep continuity is associated with fluid intelligence, or with set-shifting or mental rotation accuracy, since these are closely related to fluid intelligence (Kafadar et al., 2016; Varriale et al., 2018). The aim of the study was to investigate the relationship between sleep continuity, assessed using daily subjective sleep diaries and fluid intelligence. It was hypothesised that sleep continuity would be positively associated with 1) fluid intelligence, 2) mental rotation and 3) set-shifting.

Methods A total of 39 healthy good sleepers completed a daily subjective sleep diary (Consensus Sleep Diary-M) for

\section{Abstract 46 Table 1}

\begin{tabular}{|c|c|c|c|c|c|c|c|c|c|c|}
\hline \multicolumn{5}{|c|}{ Winter Data Acquisition (Feb-March 2021, lockdown, home schooling) } & \multicolumn{3}{|c|}{8 schools; $N_{\text {final }}=60$ (4 excluded $)$} & \multicolumn{3}{|c|}{ Age $14.5 ; 72 \%$ female } \\
\hline & $\begin{array}{c}\text { Online Survey } \\
\text { (nr participants) }\end{array}$ & $\begin{array}{c}\text { TMB } \\
\text { 1.Letter/Number } \\
\text { Switching } \\
\text { (nr participants) }\end{array}$ & $\begin{array}{c}\text { TMB } \\
\text { 2.Multiple } \\
\text { Object Tracking } \\
\text { (nr participants) }\end{array}$ & $\begin{array}{c}\text { TMB } \\
\text { 3.Verbal Paired } \\
\text { Associate } \\
\text { (nr participants) }\end{array}$ & $\begin{array}{c}\text { TMB } \\
\text { 4.Fast Choices } \\
\text { Test } \\
\text { (nr participants) }\end{array}$ & $\begin{array}{c}\text { TMB } \\
\text { 5. Visual Pairs } \\
\text { (nr participants) }\end{array}$ & $\begin{array}{c}\text { TMB } \\
\text { 6.Continuous } \\
\text { Concentration } \\
\text { (nr participants) }\end{array}$ & $\begin{array}{c}\text { EMAapp } \\
\text { (nr participants; } \\
3 \text { weeks) }\end{array}$ & $\begin{array}{c}\text { EMAapp } \\
\text { (nr entries } / 43)^{*}\end{array}$ & $\begin{array}{l}\text { Actigraphy } \\
\text { (3 weeks) }\end{array}$ \\
\hline Pre questionnaire & $56(91 \%)$ & $49(81 \%)$ & $46(72 \%)$ & $46(72 \%)$ & $46(72 \%)$ & $46(72 \%)$ & $45(70 \%)$ & \multirow{2}{*}{$57(95 \%)$} & \multirow{2}{*}{$19(44 \%)$} & \multirow[b]{2}{*}{ Upcoming } \\
\hline Post questionnaire & $47(75 \%)$ & $47(73 \%)$ & $46(72 \%)$ & $46(72 \%)$ & $39(63 \%)$ & $39(63 \%)$ & $40(64 \%)$ & & & \\
\hline \multicolumn{5}{|c|}{ Summer Data Acquisition (May-June 2021, back to school) } & 3 schools; $N_{\text {fit }}$ & inal $=15(2 \mathrm{exc}$ & cluded) & \multicolumn{3}{|c|}{ Age $14 ; 34 \%$ female } \\
\hline Pre questionnaire & $15(100 \%)$ & $15(100 \%)$ & $14(83 \%)$ & $14(83 \%)$ & $14(83 \%)$ & $14(83 \%)$ & $14(83 \%)$ & \multirow{2}{*}{$11(73 \%)$} & \multirow{2}{*}{$21(49 \%)$} & \multirow{2}{*}{ Upcoming } \\
\hline Post questionnaire & $12(74 \%)$ & $12(74 \%)$ & $12(74 \%)$ & $12(74 \%)$ & $12(74 \%)$ & $12(74 \%)$ & $12(74 \%)$ & & & \\
\hline
\end{tabular}

